\title{
Primary Hydatid Cyst of the Spleen
}

\author{
Col Hariqbal Singh", Maj Sumeet Arora ${ }^{+}$
}

MJAFI 2003; 59 : 169-170

Key Words : Hydatid cyst; Spleen

\section{Introduction}

$\mathrm{T}$ he larval form of the genus Echinococcus, of which Echinococcus granulosus is the most common, causes hydatid disease. The liver and lungs are the organs most commonly involved. Splenic involvement in hydatid disease is uncommon, representing less than $2 \%$ to $3.5 \%$ of all human infestations by Echinococcus $[1,2]$. The infestation of the spleen usually takes place by arterial route after the parasite has passed through the two filters, hepatic and pulmonary. The retrograde venous route which avoids the liver and lung, is also considered [3].

\section{Case Report}

45 year old female, a known case of diabetes mellitus and hypertension controlled with medication presented with left sided headache and diminution of vision. On investigations she was diagnosed as a case of pituitary adenoma. Radical decompression of the tumour was done followed by radiotherapy; she showed good response. On 6 monthly routine follow up blood examination showed $\mathrm{Hb}$ $11.2 \mathrm{gm} \%$, TLC $7600 / \mathrm{mm}^{3}$, and DLC $\mathrm{P}_{75} \mathrm{~L}_{20} \mathrm{M}_{3} \mathrm{E}_{5}$. Chest radiograph showed rounded egg shell like calcification under the left dome of diaphragm in the splenic area about $5 \mathrm{~cm}$ in diameter (Fig-1) suggesting a hydatid cyst. On ultrasound examination of the abdomen, a single unilocular well defined spherical cystic lesion was seen in the upper pole of the spleen with bright echogenic periphery suggesting wall calcification (Fig-2). She was subjected to computerised tomographic (CT) scan abdomen which confirmed the plain film and ultrasound findings of a cystic lesion measuring $5.5 \times 5.34$ $\mathrm{cm}$ in diameter within the spleen with attenuation value near that of water (Fig-3) having wall calcification. There was no surrounding abnormality and no enhancement following intravenous contrast administration and was diagnosed as splenic hydatid cyst.

\section{Discussion}

Hydatid disease is endemic in South America, Africa, Middle East, Turkey, Southern Europe, India, New Zealand and Australia. Infestation by hydatid disease in humans most commonly occurs in the liver (55-70\%) followed by the lung (18-35\%); the two organs can be affected simultaneously in about $5-13 \%$ of cases $[4,5]$. However it can, though rare, occur in any organ primarily or in association with hepatic or pulmonary or as multiorgan hydatidosis. Splenic hydatid cysts are uncommon and are often asymptomatic as seen in this case, it was an incidental detection. However, it may present as a painful mass in the left upper abdominal quadrant or enlarged spleen with fever [3]. The likely complications can be infection, rupture in the abdominal cavity or fistulization to the colon [3]. Harefuah in 1992 described a 20 year old soldier who presented with anaphylactic shock due to rupture of a splenic echinococcal cyst induced by blunt trauma to the left chest wall and upper abdomen. The main clinical manifestations, which developed within minutes of the trauma, were high fever, pruritus, edema of the lips and eyelids, dyspnea, stridor and rhinorrhea. Eosinophilia was not present on admission but appeared 4 days later [6].

The main problem in the diagnosis of splenic hydatidosis is in differentiating it from other splenic cystic lesions, such as epidermoid cyst, pseudocyst, abscess, haematoma, and cystic neoplasm of the spleen. Hydatidosis should always be suspected, particularly in endemic areas [7]. Marginal or crumpled egg shell like calcification in the splenic area detected on abdominal or chest radiograph are the most suggestive indications of splenic hydatidosis. More definitive

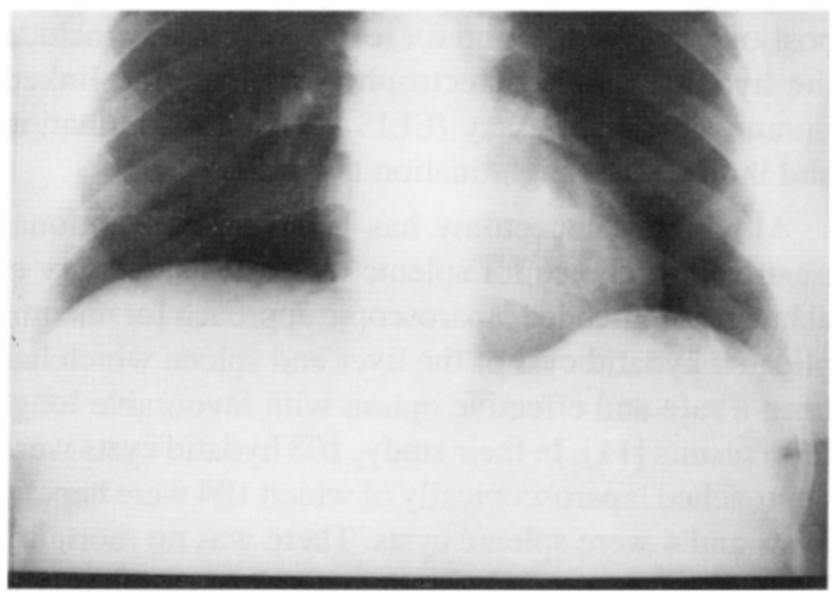

Fig. 1: Radiograph chest shows an egg shell calcification in the splenic area 


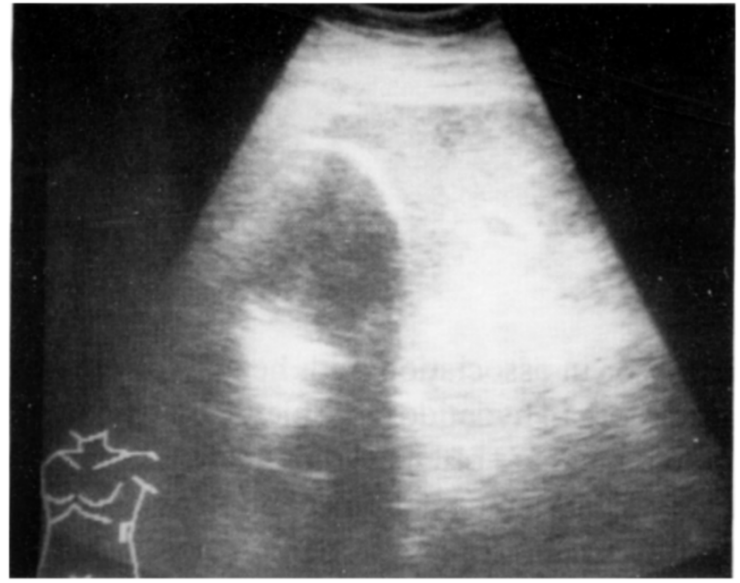

Fig. 2 : Ultrasound scan abdomen demonstrates a single unilocular well defined spherical cystic lesion in the upper pole of the spleen with bright echogenic wall.

diagnosis is based on abdominal ultrasound and CT scan which have greater sensitivity and specificity [8]. On ultrasound scan of abdomen, splenic hydatid cyst may present as a solitary unilocular or rarely multiple well defined anechoic spherical cystic lesions or may demonstrate an anechoic spherical cystic lesion with hyperechoic marginal calcification as seen in this case. CT abdomen confirms the cystic lesion with or without daughter cysts within the spleen with attenuation value near that of water and does not enhance after intravenous contrast administration. It is more sensitive than ultra sonography in depicting subtle wall calcification [9].

The hydatid cyst consists of 3 layers : an adventitia formed of compressed host tissue, a middle layer of friable ectocyst and an inner germinal layer from which large number of scolices are produced, which are the heads of developing worms. Daughter cysts are formed if the viability is threatened. Different serological tests are being carried out for the diagnosis, screening and post-operative follow-up for recurrence. These include the hydatid immunoelectrophoresis, enzyme linked immunoabsorbent assay (ELISA), latex agglutination and indirect haemaglutination test [10].

Although splenectomy has been the conventional treatment of choice for splenic hydatidosis, Khoury et al had recommended laparoscopic approach for uncomplicated hydatid cyst of the liver and spleen which has been a safe and effective option with favourable longterm results [11]. In their study, 108 hydatid cysts were approached laparoscopically of which 104 were hepatic cysts and 4 were splenic cysts. There was no mortality [11]. However, pre and post operative one month course of Albendazole and two week course of Praziquantel should be considered in order to sterilize the cyst, decrease the chance of anaphylaxis, decrease the tension in the cyst wall, thus reducing the risk of spillage during surgery and to reduce the recurrence rate post

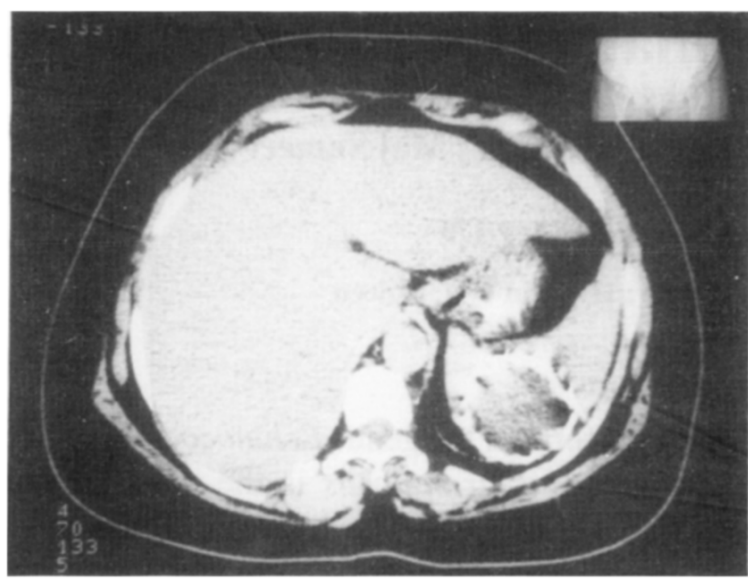

Fig. 3: CT scan showing a well defined cystic lesion with calcified wall in the upper pole of the spleen.

operatively $[7]$.

Splenic involvement is an uncommon manifestation of hydatid disease and should be suspected on detection of any splenic cyst especially in endemic areas of the world. Ultrasonography and CT are the most useful diagnostic tools.

\section{References}

1. Franquet T. Montes M. Lecumberri FJ. Esparza J. Bescos JM. Hydatid disease of the spleen : imaging findings in nine patients. Am J Roentgenol 1990;154:525-8.

2. Uriarte C. Pomares N. Martin M. Conde A. Alonso N. Bueno A. Splenic hydatidosis. Am J Trop Med Hyg 1991:44:420-3.

3. Ionescu A, Jakab A. Jutis T, Forai F, Ota A. Splenic hydatid cyst (Article in Romanian). Rev Med Chir Soc Med Nat lasi. 1990;94:525-8.

4. Kir A, Baran E. Simultaneous operation for hydatid cyst of right lung and liver. Thorac Cardiovasc Surgeon 1995;43:624.

5. Guntz M, Coppo B, Lorimier G, Cronier P. Hydatid cyst of the liver appearing late (10-22 years) after surgical treatment of pulmonary hydatidosis - physiopathologic problems. J Chir Paris 1990;27:375-81.

6. Bitton M, Kleiner-Baumgarten A, Peiser J, Barki Y, Sukenik S. Anaphylactic shock after traumatic rupture of a splenic echinococcal cyst (article in Hebrew). Harefuah 1992;122:2268.

7. Goel MC, Agarwal MR, Misra A. Percutaneous drainage of renal hydatid cyst : early results and follow-up. Br J Urol 1995:75:724-8.

8. Herrera Merino N, Abascal Morte J, Diaz del Rio Botas M et al. Splenic hydatid cyst. A report of 16 cases (Article in Spanish). Ev Esp Enferm Dig 1991:79:254-8.

9. Kalouidouris A. Pissilosis C. Pontifex G. CT characterization of multivesicular hydatid cysts. J Comput Assist Tomogr 1984:8:839-45.

10. Kune GA. Morris DI. Hydatid disease. In : Schwasyz \& Ellis, editors. Maingot's Abdominal Operations, $9^{\text {th }}$ ed Appleton \& Lange, 1989:1225-40.

11. Khoury G, Abiad F, Geagea T, Nabout G. Jabbour A. Laparoscopic treatment of hydatid cysts of the liver and spleen. Surg Endosc 2000;14:243-5. 